\title{
Isothermal kinetic of phase transformation and mixed electrical conductivity in $\mathrm{Bi}_{3} \mathrm{NbO}_{7}$
}

\author{
X. P. Wang ${ }^{\text {a, }}{ }^{\text {, G. }}$ Corbel $^{\text {a }}$, S. Kodjikian ${ }^{\text {a }}$, Q. F. Fang ${ }^{\text {b }}$, and P. Lacorre ${ }^{a,{ }^{*}}$ \\ ${ }^{a}$ Laboratoire des Oxydes et Fluorures - UMR CNRS 6010, Université du Maine, \\ Avenue Olivier Messiaen, 72085 Le Mans Cedex 9, France \\ ${ }^{\mathrm{b}}$ Key Laboratory of Materials Physics, Institute of Solid State Physics, \\ Chinese Academy of Sciences, 230031 Hefei, P. R. China
}

\begin{abstract}
Bismuth niobate $\left(\mathrm{Bi}_{3} \mathrm{NbO}_{7}\right)$ exists under two crystallographic modifications, a tetragonal (type-III) phase between 800 and 900 degrees C, and a pseudocubic (type-II) phase above and below this thermal range. The quenching at room temperature of pseudocubic type-II phase made it possible to carry out a detailed study of the transformation kinetics of this metastable type-II phase to the stable type-III phase, using isothermal in situ X-ray diffraction. The obtained Avrami exponent and activation energy for the transition are around 2.5 and $3.25 \mathrm{eV}$, respectively. The value of the Avrami exponent is consistent with a three-dimensional diffusion-controlled transformation with constant nucleation rate. Investigations of electrical properties using AC impedance spectroscopy and Wagner polarization method show that the tetragonal phase exhibits higher ionic and electronic conductivities than those of the pseudocubic form. Such a deviation is likely to originate from different distributions of cations/electronic-lone-pairs and oxygen vacancies.
\end{abstract}

Keywords : bismuth niobium oxide; metastability; solid state diffusion-controlled phase transition; Avrami exponent; thermal volume expansion; fast oxide-ion conduction; electronic conductivity; ionic transference number; X-ray powder diffraction; impedance spectroscopy

\section{Introduction}

The high-temperature form of bismuth oxide $\delta-\mathrm{Bi}_{2} \mathrm{O}_{3}$, which has an oxygen-deficient fluorite-type structure, was recognized as one of the best solid-state oxide ion conductors owing to the high concentration of intrinsic oxygen vacancies [1-3]. Although the high-temperature cubic phase only exists in the temperature range from $730{ }^{\circ} \mathrm{C}$ up to its melting point $825^{\circ} \mathrm{C}$ and cannot be quenched to room temperature, the stability of the high conductivity phase can be greatly enhanced by partial substitution of bismuth with trivalent rare earth (such as $\mathrm{Gd}, \mathrm{Y}, \mathrm{Er}$ ions) or with higher-valency cations (such as $\mathrm{Nb}$, $\mathrm{Ta}$, or W) [4-8]. Such cation substitutions generally form different solid solutions with good ionic conductivity in combination with electronic conductivity. This mixed ionic and electronic conduction property makes them suitable for use in electrochemical devices such as high-purity

${ }^{*}$ Corresponding author. Fax: +33243833506 .

E-mail address: Philippe.Lacorre@univ-lemans.fr (P. Lacorre). oxygen generators, electrochemical sensors and anode materials in solid oxide fuel cells $[9,10]$.

Among the many choices of substituting cations in $\delta$ $\mathrm{Bi}_{2} \mathrm{O}_{3}, \mathrm{Nb}^{5+}$ is probably the most frequently used one owing to its high efficiency to stabilize the cubic phase to room temperature. The minimum concentration of niobium oxide necessary to stabilize $\delta-\mathrm{Bi}_{2} \mathrm{O}_{3}$ is $10 \mathrm{~mol} \%$ [8]. Studies of the $\mathrm{Bi}_{2} \mathrm{O}_{3}-\mathrm{Nb}_{2} \mathrm{O}_{5}$ phase diagram revealed that it contains at least four distinct phases (types I-IV) in the composition range between pure $\mathrm{Bi}_{2} \mathrm{O}_{3}$ and $5 \mathrm{Bi}_{2} \mathrm{O}_{3}: 3 \mathrm{Nb}_{2} \mathrm{O}_{5}$ [11-13]. For the type-II phase that exists in the composition range from $6.2 \%$ to $25 \% \mathrm{Nb}_{2} \mathrm{O}_{5}$ [13], it crystallizes with a three-dimensional (3D) incommensurate modulated superstructure of a fluorite-type cubic subcell [12]. A commensurate structural model derived from the formalism of superspace symmetry predicts interpenetrating $\mathrm{Nb}$ rows at $60^{\circ}$ to one another, with a specific distribution of oxygen vacancies [14]. Type-III single phase with $25 \% \mathrm{Nb}_{2} \mathrm{O}_{5}$ was initially synthesized at $900^{\circ} \mathrm{C}$ by Ling et al. $[13,15]$. In contrast to the incommensurate type-II phase, the type-III modification has a commensurate superstructural ordering 
relative to a tetragonal subcell [16]. The structure of this type-III form was modeled as a tetragonal deficient fluorite-type structure with an $I$-centered $3 \times 3 \times 7$ supercell $[7,15]$. This superstructure consists of independent rows and isolated tetrahedral units of corner-sharing $\mathrm{NbO}_{6}$ octahedra imbedded in a $\mathrm{Bi}-\mathrm{O}$ matrix, and can be considered as a hybrid of fluorite and pyrochlore structural types [15].

Previous studies have shown that the type-II phase is not stable between 800 and $900{ }^{\circ} \mathrm{C}$ at which it undergoes a phase transition to the tetragonal type-III form $[16,17]$. Contrary to an earlier claim of a stability range from room temperature to melting point $1100^{\circ} \mathrm{C}$ [13], the type-III phase is stable only below $900{ }^{\circ} \mathrm{C}$. Above $930^{\circ} \mathrm{C}$, it transforms back to a single type-II modification after passing a mixed tetragonal-pseudocubic phase regime between 900 and $930{ }^{\circ} \mathrm{C}$ [17]. The formation characteristics and stability range of the type-III phase were investigated by Pirnat et al. [18], who studied the type-II/type-III phase transition kinetics through room temperature X-ray powder diffraction. They observed a temperature-dependent induction time for the phase transformation, and were able to determine the Avrami exponent to be around 1.5.

In this paper, we reexamine the kinetic process of this $\mathrm{Bi}_{3} \mathrm{NbO}_{7}$ phase transition in real isothermal conditions, using in situ X-ray powder thermodiffraction, and find significantly different results. In addition, the effects of structural difference on the ionic and electronic electrical properties of both phases are investigated by AC impedance spectroscopy and Wagner polarization method.

\section{Experimental}

Polycrystalline $\mathrm{Bi}_{3} \mathrm{NbO}_{7}$ was prepared by conventional solid-state reaction from a stoichiometric mixture of highpurity $\mathrm{Bi}_{2} \mathrm{O}_{3}(99.9 \%)$ and $\mathrm{Nb}_{2} \mathrm{O}_{5}(99.9 \%)$ powder. After having been well mixed in an agate mortar, the initial powders were heated at $700^{\circ} \mathrm{C}$ for $5 \mathrm{~h}$ at first, to form lessvolatile $\mathrm{Nb}$ compounds and avoid $\mathrm{Bi}_{2} \mathrm{O}_{3}$ loss in the following sintering process at higher temperature. The pre-reacted powder was then calcined at $800^{\circ} \mathrm{C}$ for $5 \mathrm{~h}$, and finally obtained as a homogenous type- $\mathrm{II}_{3} \mathrm{Bi}_{3} \mathrm{NbO}_{7}$ (labeled as " $\delta-\mathrm{BN}_{\mathrm{LT}}$ powder") for the kinetic studies of the pseudocubic-tetragonal transformation. In the studies of electrical properties, two series of pellets with tetragonal and pseudocubic modifications were prepared. The powders with pure type-III and type-II forms were obtained by annealing the $\delta$ - $\mathrm{BN}_{\mathrm{LT}}$ powder at $870{ }^{\circ} \mathrm{C}$ for $12 \mathrm{~h}$ and $950^{\circ} \mathrm{C}$ for $6 \mathrm{~h}$, respectively. The obtained powder samples were shaped in a uniaxial press at first, and then pressed in an isostatic Top Industrie press (about $0.55 \mathrm{GPa}$ ). In order to get high-density ceramic pieces, the tetragonal and the pseudocubic samples were finally fired for $5 \mathrm{~h}$ at 870 and $950{ }^{\circ} \mathrm{C}$, respectively. The density of both pellets was about $97 \%$ of their theoretical density calculated from X-ray diffraction (XRD) data.
XRD patterns were recorded on a $\theta / \theta$ Bragg-Brentano X'pert MPD PRO diffractometer ( $\mathrm{Cu} K \alpha_{1+2}$ radiations) equipped with an X'celerator detector and a HTK 1200 Anton Paar chamber. The high-resolution diffratograms were collected in the $\left[5^{\circ}-130^{\circ}\right]$ scattering angle range with a step of $0.0167^{\circ}$ and scanning time of $200 \mathrm{~s}$ per step. Based on a standard fluorite prototype cell, the whole pattern matching refinement of powder XRD patterns was performed using the Fullprof program [19]. For the kinetics study, each scan was performed with a step of $0.0167^{\circ}$ in the $2 \theta$ range $\left[54.5^{\circ}-57^{\circ}\right]$, where the cubic diffraction line (311) and two tetragonal diffraction lines $(113,131)$ are wholly covered at each experimental temperature. In each isothermal experiment, the sample was continuously scanned in terms of successive periods of $10 \mathrm{~min}$ for 6-24 h, depending on the annealing temperature. The integrated intensity of the three peaks was determined using the fit profile option of X'Pert Highscore Plus program (version 2.0a).

The electron diffraction study was performed with a $200 \mathrm{kV}$ JEOL 2010 TEM equipped with a side entry $\pm 30^{\circ}$ double tilt specimen holder. The sample was prepared by grinding raw powder in ethanol and depositing one droplet on a carbon-coated holey film.

For the measurements of charge transport, thin platinum films were deposited as electrodes by magnetron sputtering on both flat faces of the pellet samples. The impedance data were collected on an Schlumberger Solartron SI 1260 frequency response analyser working with a Dielectric Interface Solartron 1296 in the frequency range of $0.05-10 \mathrm{MHz}$. The electronic conductivity was measured by Wagner polarization method on an Schlumberger Solartron 1287/1260 electrochemical response analyser, in which the platinum films were used as the ion-blocking electrodes.

\section{Results and discussion}

\section{$X$-ray diffraction at different temperatures and electron diffraction}

Fig. 1 presents the XRD patterns of $\mathrm{Bi}_{3} \mathrm{NbO}_{7}$ recorded at four typical temperatures of $25,760,870$ and $950{ }^{\circ} \mathrm{C}$. The initial sample selected is the $\delta$ - $\mathrm{BN}_{\mathrm{LT}}$ powder. Before scanning at 870 and $950^{\circ} \mathrm{C}$, the sample was annealed for $6 \mathrm{~h}$ at each given temperature in order to get single type-III and type-II phases, respectively. In Fig. 1, only a portion of the $2 \theta$ range is given for clarity. The three patterns recorded at 25,760 and $950{ }^{\circ} \mathrm{C}$ have the same appearance. The basic lines correspond to those of a cubic fluorite phase, and the superstructure peaks (labeled by stars in Fig. 1) are consistent with the so-called Type-II phase. The modulation parameter $\varepsilon(\sim 0.383)$ is consistent with the reported values in similar $\left(\mathrm{Bi}_{1-x} \mathrm{Nb}_{x}\right)_{2} \mathrm{O}_{3+2 x}$ phases [13]. It does not evolve with temperature in the low-temperature stability domain ( $\varepsilon$ around 0.3835 up to $800^{\circ} \mathrm{C}$ ) but decreases slightly at higher temperature $(\varepsilon=0.3821$ at 


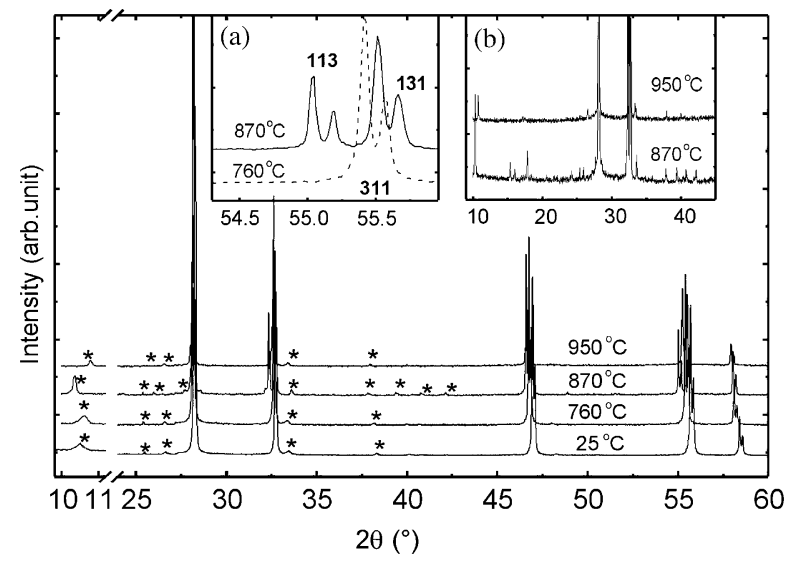

Fig. 1. Typical X-ray diffraction patterns of $\mathrm{Bi}_{3} \mathrm{NbO}_{7}$ recorded at various temperatures. Inset (a) enlargement showing the (311) cubic and $(113,131)$ splitted tetragonal diffraction lines (single subcell indexation); (b) on a larger scale, satellite reflections recorded at $870{ }^{\circ} \mathrm{C}$ (type-III) and $950{ }^{\circ} \mathrm{C}$ (type-II).

$950{ }^{\circ} \mathrm{C}$ ). It is the only noticeable difference between the low and high temperature type-II phases. Note that this $\varepsilon$ value is practically identical to that measured on the $x=0.234$ composition $(\varepsilon=0.384)$ [13], thus confirming that $\varepsilon$ is nearly constant outside the range $0.13 \leqslant x \leqslant 0.24$ [20].

In contrast to the previous phases, a significant tetragonal splitting is observed in the pattern collected at $870^{\circ} \mathrm{C}$, more distinctly visible in inset (a) of Fig. 1. Both splitting and superstructure peaks correspond to the typeIII $3 \times 3 \times 7$ tetragonal form of $\mathrm{Bi}_{3} \mathrm{NbO}_{7}$.

The structural transitions detected in our X-ray thermodiffractograms are in good agreement with Valant et al. investigations [16,17]. In Fig. 2, the thermal evolutions of the lattice parameters and volume of type-II (a) and typeIII (b) subcells from room temperature to $950{ }^{\circ} \mathrm{C}$ are presented. The two samples used for this study were the original $\delta-\mathrm{BN}_{\mathrm{LT}}$ powder as the initial type II phase (Fig. 2a), and a sample annealed at $870{ }^{\circ} \mathrm{C}$ for $6 \mathrm{~h}$ as the initial type III phase (Fig. 2b). In between two successive thermal steps, a heating rate of $10 \mathrm{~K} / \mathrm{min}$ was used, and at each given temperature, the sample was thermally stabilized for $20 \mathrm{~min}$ before XRD scanning. Each XRD diffractogram was collected in the $\left[5^{\circ}-130^{\circ}\right]$ scattering angle as described in the experimental section. In Fig. 2(a), the unequal values of $a$ and $c$ evidence the presence of the tetragonal form between 800 and $900{ }^{\circ} \mathrm{C}$. It is to be noted that, compared with the extrapolated value for the pseudocubic form (the dotted line in Fig. 2(a)), the subcell volume of the tetragonal phase is a little smaller at comparable temperatures, due to either a slight shrinkage or a levelling off at the pseudocubic-tetragonal transformation. Fig. 2(b) shows the thermal evolution of the type-III tetragonal cell with transition to type-II pseudocubic cell around $900{ }^{\circ} \mathrm{C}$. Note that the cell volume is slightly smaller than that of the cubic cell in the same thermal range. At low temperature, the stable phase seems to be the tetragonal one, whereas the pseudocubic phase is meta-

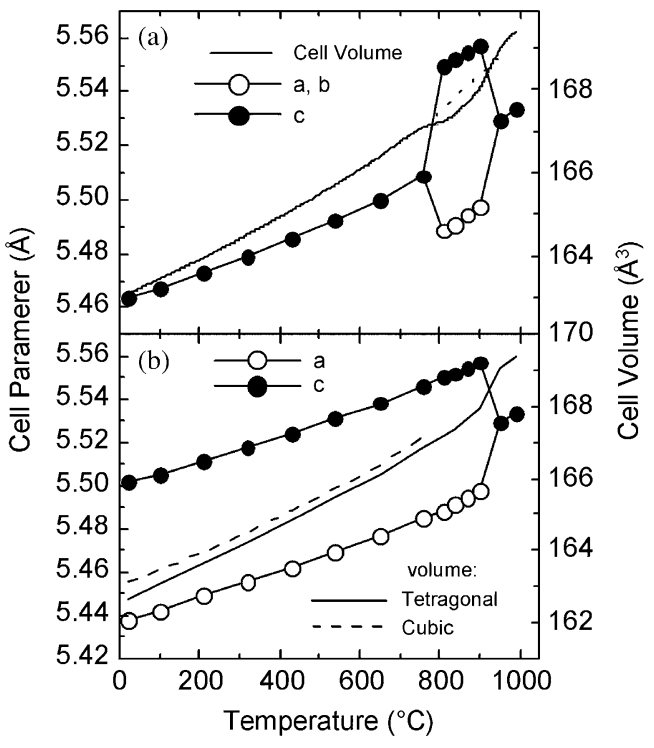

Fig. 2. Thermal evolution of lattice parameter and unit cell volume of $\mathrm{Bi}_{3} \mathrm{NbO}_{7}$ upon heating: (a) pseudocubic type-II phase, showing the transition to tetragonal type-III phase between 800 and $900{ }^{\circ} \mathrm{C}$; (b) tetragonal type-III phase, showing the transition to pseudocubic type-II phase above $900^{\circ} \mathrm{C}$.

stable (stabilized from quenching the high-temperature form) and transforms to the stable tetragonal phase upon heating.

In order to further characterize both forms and confirm the presence of type-II and type-III superstructures, electron diffraction patterns were collected on samples annealed at 800 and $870{ }^{\circ} \mathrm{C}$, respectively. For the pseudocubic type-II form, according to Ling et al. [13], any Bragg reflection $H$ can be written in the form

$$
\begin{aligned}
H= & h \mathrm{a}_{\mathrm{f}}^{*}+k \mathrm{~b}_{\mathrm{f}}^{*}+l \mathrm{c}_{\mathrm{f}}^{*}+m \varepsilon \mathrm{a}_{\mathrm{f}}^{*} \\
& +n \varepsilon \mathrm{b}_{\mathrm{f}}^{*}+p \varepsilon \mathrm{c}_{\mathrm{f}}^{*}[=h l k m n p]^{*} .
\end{aligned}
$$

Indeed, selected area electron diffraction (SAED) patterns present main diffraction spots which are singly indexed $[h k l]$ onto the $\delta-\mathrm{Bi}_{2} \mathrm{O}_{3}$-like fluorite cubic subcell, as shown in Fig. 3(a) and (c). In addition, satellite spots typical of the type-II structure are observed, in agreement with the electron diffraction study by Tang and Zhou [12] on another composition of the $\mathrm{Bi}_{2-x} \mathrm{Nb}_{x} \mathrm{O}_{3+x}$ solid solution. In the outlines of Fig. 3(b) and (e) enlarged from 3(d), the satellite spots of main central spot $=[000 \mathrm{mnp}]$ are indexed as $m n p$ for simplicity. The modulation parameter $\varepsilon$ measured from the satellite spots position is $0.38(1)$, in agreement with the value measured by XRD. Note that extra spots are also observed on the Fig. 3(a) SAED pattern, as in all observed grains along the [001] axis, showing that they are in fact multi-domains crystallites. In other directions however, like along [110], crystals appear as mono-domains (Fig. 3(c)).

For the tetragonal type-III form prepared at $870^{\circ} \mathrm{C}$, all the crystallites exhibit a $3 \times 3 \times 7$ tetragonal superstructure of the fluorite-type $\delta-\mathrm{Bi}_{2} \mathrm{O}_{3}$. Typical [100] and [001] SAED patterns are given in Fig. 4(a) and (b), respectively. They 

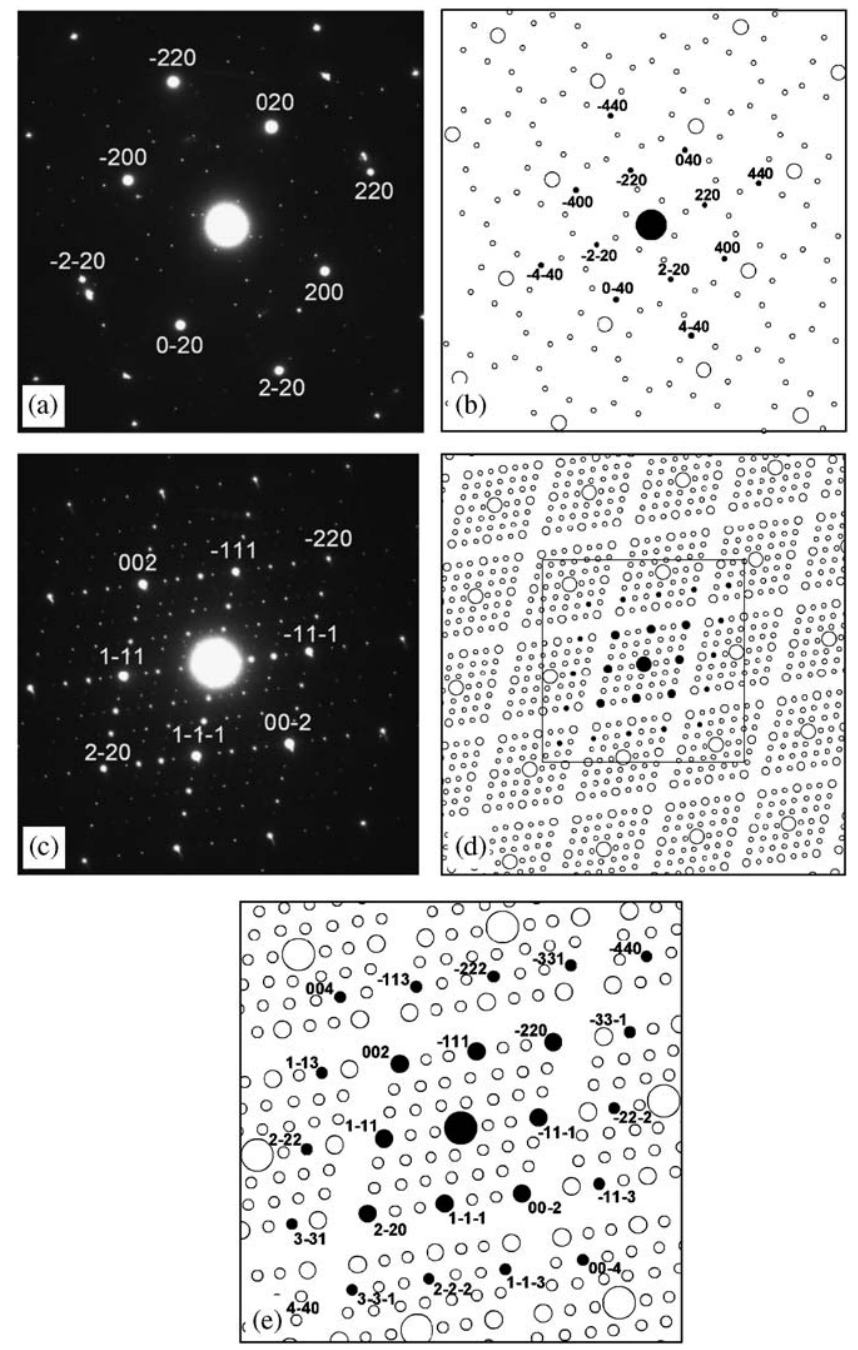

Fig. 3. Typical SAED patterns of $\mathrm{Bi}_{3} \mathrm{NbO}_{7}$ type-II phase along [001] (a) and $[110]$ (c), together with their outlines (b) and (d), respectively) Indexation of the superstructure reflections are given in (b) and (e), which is an enlargement of (d). For clarity, the three first main $h k l$ indices only are given on the photographs for main reflections [hkl000], and the three last $m n p$ indices only on the outlines for satellites of the central spot [000mnp] (full circles).
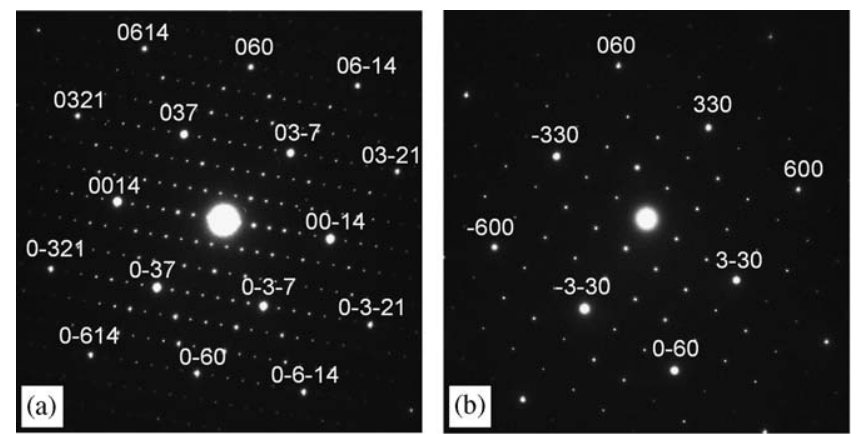

Fig. 4. SAED patterns of $\mathrm{Bi}_{3} \mathrm{NbO}_{7}$ type-III phase along [100] (a) and [001] (b).

evidence the I-type condition of reflection, which is in agreement with the type-III superstructure determined by Ling and Johnson [15].

\section{Kinetics of the pseudocubic-tetragonal phase transformation}

Since the high temperature pseudocubic type-II form can be readily preserved to lower temperatures as a metastable phase by quenching, it is possible to carry out a detailed study of the transformation kinetics of the metastable pseudocubic phase to the stable tetragonal phase.

\section{Isothermal in situ XRD measurements}

The isothermal kinetics of the pseudocubic-tetragonal phase transition in $\mathrm{Bi}_{3} \mathrm{NbO}_{7}$ has been investigated systematically using in situ X-ray thermodiffraction. In order to timely detect the evolution of phase transformation, a scanning period of $10 \mathrm{~min}$ is chosen for XRD measurements.

For the kinetic investigation of low-temperature pseudocubic-tetragonal transformation, four different annealing temperatures of $810,840,870$ and $900^{\circ} \mathrm{C}$ were selected. The initial powders before annealing at each given temperature are identical type-II $\delta$ - $\mathrm{BN}_{\mathrm{LT}}$ samples. In Fig. 5, a selection of diffraction patterns recorded during the annealing at $870{ }^{\circ} \mathrm{C}$ for various times are presented. At $t=0$ the cubic line (311) only is detected (indices in the single subcell, $a \sim 5.5 \AA$ ), indicating the presence of the initial type-II $\mathrm{Bi}_{3} \mathrm{NbO}_{7}$ phase only. After a while, two diffraction lines $(113,131)$ associated with the tetragonal phase appear (single subcell indices). The coexistence of the three diffraction lines $(311,113,131)$, hence of the two phases, is visible for about $2 \mathrm{~h}$ at this temperature (Fig. 5(b)-(f)). The intensity of the tetragonal diffraction lines gradually increases, while that of the cubic line decreases with time. At $t=180 \mathrm{~min}$, only the tetragonal lines are visible (Fig. 5(g)), indicating the completion of the

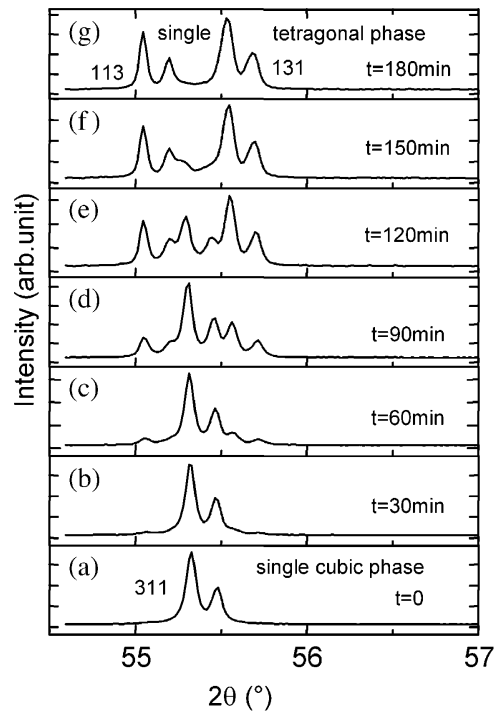

Fig. 5. Evolution with time of XRD patterns associated with the pseudocubic-tetragonal transition of $\mathrm{Bi}_{3} \mathrm{NbO}_{7}$ recorded at $870{ }^{\circ} \mathrm{C}$. Pattern (a) single pseudocubic phase; Patterns (b)-(f) a two-phase mixture of pseudocubic and tetragonal forms; Pattern (g) single tetragonal phase. 
pseudocubic-tetragonal transformation. For the other isothermal experiments, similar processes are observed, with a slower or faster evolution depending on the annealing temperature.

The transformation rate $\alpha$ at any time $t$ can be calculated from the growth of the new Bragg reflection $(h k l)$ as defined by [21]

$\alpha(t)=\frac{I_{h k l}(t)}{I_{h k l}(\infty)}$,

where $I_{h k l}(t)$ represents the integrated intensity of reflection $(h k l)$ at time $t$ and $I_{h k l}(\infty)$ is the integrated intensity when the reaction is complete. Here, the growth of the tetragonal line (113) is used as the indicator. The plots of transformation rate $\alpha(t)$ versus time $t$ at different annealing temperatures is given in Fig. 6, in which a similar tilted " $S$ " shape kinetic curve based on nucleation and domain growth is observed in each isothermal transformation. With increasing annealing temperature, the reaction time is found to sharply decrease from around $1440 \mathrm{~min}$ at $810^{\circ} \mathrm{C}$ to $120 \mathrm{~min}$ at $900^{\circ} \mathrm{C}$. In the phase transformation investigation reported by Pirnat et al. [18], a long induction time about $126-285 \mathrm{~min}$ is observed in the $\mathrm{Bi}_{3} \mathrm{NbO}_{7}$ samples initially fired at $700{ }^{\circ} \mathrm{C}$. However, we did not observe any induction time in our samples. The reason for this discrepancy might be ascribed to the difference either in the thermal procedure or in the samples shaping (pellets in reference 18, powders in this work). In Fig. 7, the fullwidth at half-maximum (FWHM) as a function of time for the diffraction patterns are given. A decrease of FWHM for the tetragonal line and an increase of FWHM for the cubic line are observed in each isothermal transformation. Since the peak width is related to the coherent domain size, this evolution reflects the progressive increase of the typeIII domains (decreasing width) at the expense of the type-II domains (increasing width).

For the kinetics of the higher-temperature tetragonalpseudocubic transition, diffraction patterns were recorded above $930^{\circ} \mathrm{C}$, since a mixture of type-II and type-III phases is always observed between 900 and $930^{\circ} \mathrm{C}$ [17]. Using the

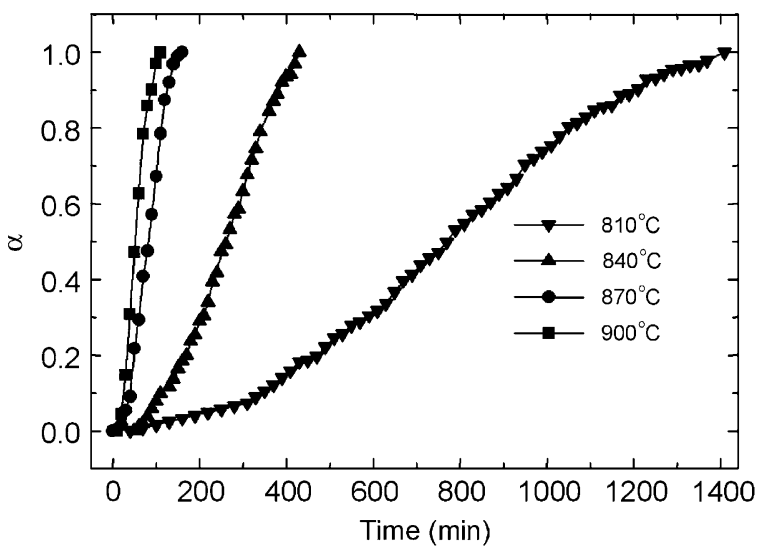

Fig. 6. Time dependence of the transformation rate of type-II $\mathrm{Bi}_{3} \mathrm{NbO}_{7}$ into the type-III phase at four different temperatures.

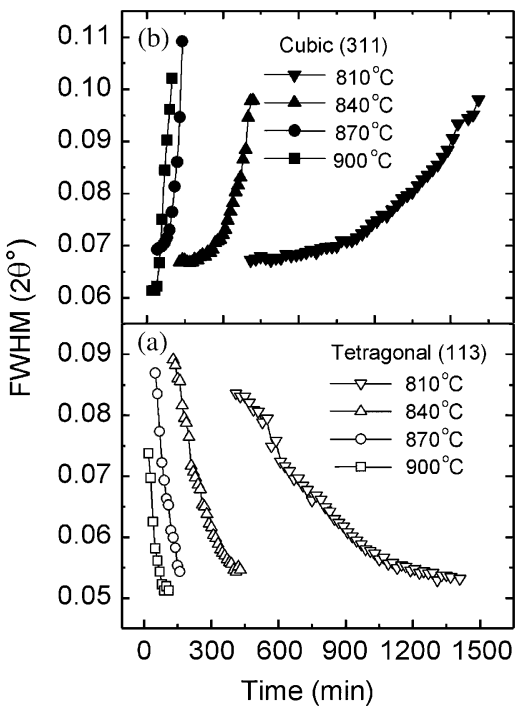

Fig. 7. Evolution with time of the full-width at half-maximum (FWHM) of tetragonal line (113) and cubic line (311) at four different temperatures.

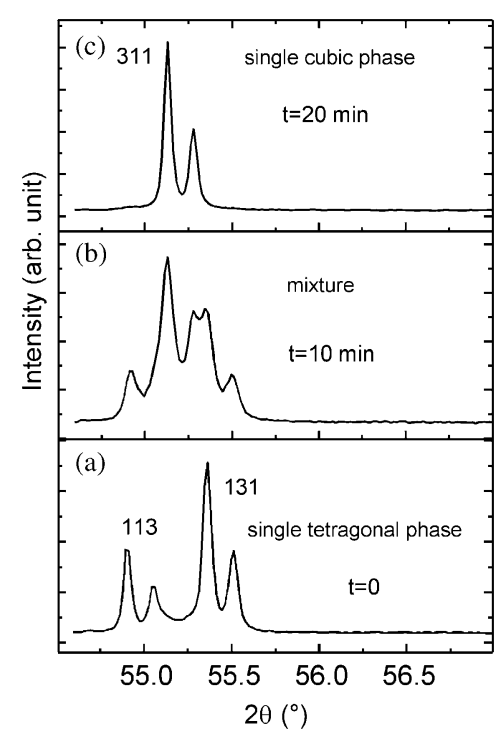

Fig. 8. Evolution with time of XRD patterns associated with the tetragonal to pseudocubic transition at $940^{\circ} \mathrm{C}$. Pattern (a): single tetragonal phase; Pattern (b): a two-phase mixture of pseudocubic and tetragonal forms; pattern (c): single pseudocubic phase.

same experimental procedure mentioned above, an isothermal scanning at $940{ }^{\circ} \mathrm{C}$ was performed, as shown in Fig. 8. From the single tetragonal phase to a single pseudocubic phase, the total reaction time is found to be limited to $20 \mathrm{~min}$, implying that the nucleation and growth of the higher-temperature tetragonal-pseudocubic transformation occurs with a faster kinetic than the pseudocubic-tetragonal transformation.

\section{Avrami parameters and activation energy of the phase transformation}

The isothermal reaction kinetics of solid-solid phase transitions are usually expressed by the well-known 
Johnson-Mehl-Avrami (JMA) equation [22-24]:

$\alpha(t)=1-\exp \left(-(k t)^{n}\right)$

leading to

$\ln (-\ln (1-\alpha(t)))=n \cdot \ln (k)+n \cdot \ln (t)$,

where $\alpha(t)$ is the transformation rate mentioned in the above section, $n$ is the Avrami exponent and $k$ is the reaction rate constant that includes nucleation and growth. In the reaction temperature regime, the temperature dependence of parameter $k$ can be expressed by an Arrhenius-type relation:

$k=k_{0} \exp \left(-\frac{E}{R T}\right)$,

where $k_{0}$ is the frequency factor, $T$ is the absolute temperature, $R$ is the gas constant and $E$ is the effective activation energy that describes the overall transition process.

Fig. 9 gives the Avrami plots of the pseudocubictetragonal transformation in $\mathrm{Bi}_{3} \mathrm{NbO}_{7}$ at the four annealing temperatures of $810,840,870$ and $900{ }^{\circ} \mathrm{C}$. It can be seen that a linear relationship between $\ln (-\ln (1-\alpha))$ and $\ln (t)$ is observed, demonstrating that the phase transition process can be adequately described by the JMA relation. Through the least-squares linear fitting of Eq. $\left(2^{\prime}\right)$, the kinetic parameters $n$ and $k$ can be deduced from the slope and the intercept of the $\ln (-\ln (1-\alpha))$ versus $\ln (t)$ plots, as summarized in Table 1. With increasing annealing temperature from 810 to $900{ }^{\circ} \mathrm{C}$, the kinetic parameter $n$ slightly increases from 2.46 to 2.62 (indicating that the annealing temperature has a little influence on the growth mechanism [22-24]) whereas $k$ varies from $1.90 \times 10^{-5}$ to $2.5 \times 10^{-4} \mathrm{~s}^{-1}$.

The activation energy and frequency factor of the pseudocubic-tetragonal transformation can be calculated by taking the logarithm of both sides of Eq. (3):

$\ln (k)=\ln \left(k_{0}\right)-E / R T$.

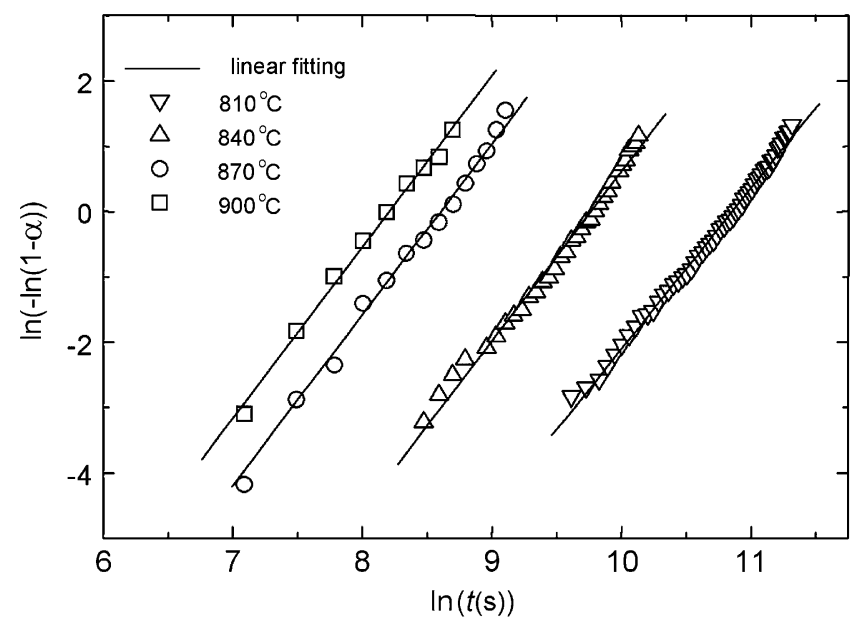

Fig. 9. Avrami plots for the isothermal pseudocubic-tetragonal transformation in $\mathrm{Bi}_{3} \mathrm{NbO}_{7}$ at four different temperatures.
Table 1

The Avrami kinetic parameters of $n$ and $k$ as a function of isothermal annealing temperature for the pseudocubic-tetragonal transformation in $\mathrm{Bi}_{3} \mathrm{NbO}_{7}$ compound

\begin{tabular}{llr}
\hline Annealing temperature $\left({ }^{\circ} \mathrm{C}\right)$ & $n$ & $-\ln (k)$ \\
\hline 810 & 2.46 & 10.87 \\
840 & 2.60 & 9.78 \\
870 & 2.61 & 8.49 \\
900 & 2.62 & 8.20 \\
\hline
\end{tabular}

Fig. 10 shows the dependence of $\ln (k)$ on $1 / T$ for the transition process in $\mathrm{Bi}_{3} \mathrm{NbO}_{7}$. From the slope and the intercept of fitting line, the activation energy $E$ and the frequency factor $k_{0}$ are determined as $3.25 \mathrm{eV}$ and $2.7 \times 10^{10} \mathrm{~s}^{-1}$, respectively.

Both $n$ and $k$ parameters are at variance from those measured by Pirnat et al., who found an Avrami exponent around 1.5 , and a $k$ parameter independent of temperature [18].

The Avrami exponent can be expressed $[25,26]$ in terms of nucleation and growth parameters, $n=a+b c$, where

$a$ is the nucleation index which governs the time dependence of the number of nuclei per unit volume of untransformed material $N \propto t^{a}$ ( $a=0$ for nucleation rate 0 , $a=1$ for constant nucleation rate, $a>1$ for increasing and $0<a<1$ for decreasing nucleation rates, respectively).

$b$ is the dimensionality of the growth $(b=1,2,3$ for $1 \mathrm{D}$, 2D, 3D growth, respectively),

$c$ is a growth index depending on the type of transformation (typically $c=1$ for a linear growth, as in interface-controlled growth, and $c=1 / 2$ for a parabolic growth, as in diffusion-controlled growth).

The point here is to determine the types of nucleation and growth which prevail in our case. The transition taking place between two phases with same compositions, which are modifications of the same structural type, one would be tempted to consider it an interface-controlled transition. However, one phase is commensurate while the other is not, and moreover the $\mathrm{Bi} / \mathrm{Nb}$ cations ordering is different, with $\mathrm{Nb}$ in interpenetrated strings at $60^{\circ}$ to one another in one phase (type-II), and in independent strings at $90^{\circ}$ to one another in the other (type-III). It shows that the phase transformation cannot simply proceed through a mere slight shift of interface atoms as in interface-controlled growth, but has to occur through a longer-range migration of both anions and cations as in diffusion-controlled growth $(c=1 / 2)$. Then the simplest way to explain the $n=2.5$ Avrami exponent is to consider that there is a constant nucleation rate during transformation $(a=1)$ and that growth is 3 -dimensional $(b=3)$.

There is a discrepancy, however, between the $n=2.5$ Avrami exponent measured in this study and the $n=1.5$ value found in the previous work by Pirnat et al. [18]. Indeed there are major differences between the two experiments, since Pirnat et al. study pellets first annealed 


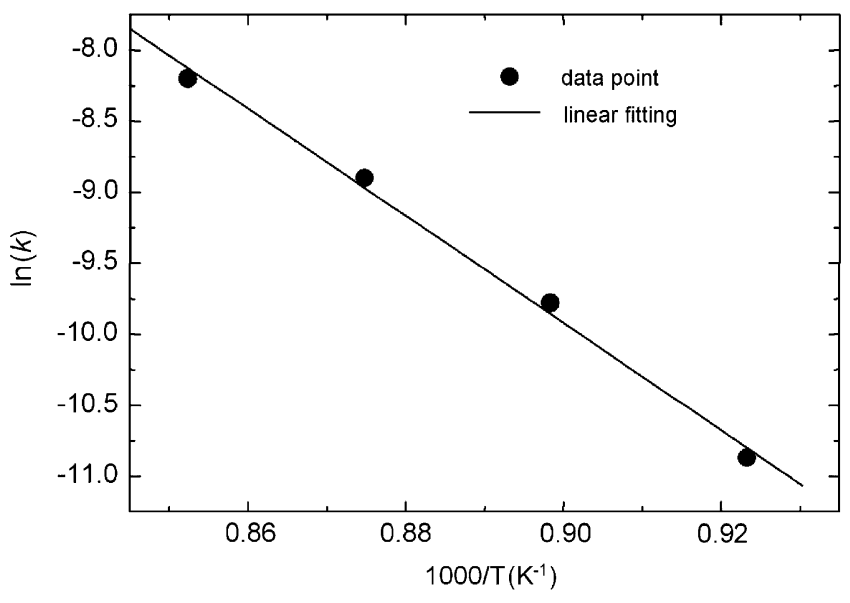

Fig. 10. Arrhenius plot for the isothermal $\mathrm{Bi}_{3} \mathrm{NbO}_{7}$ pseudocubictetragonal phase transformation.

then cooled down (not really isothermal conditions), while we are studying the isothermal kinetics of powders. General comments and comparisons have already been made on kinetics studies derived from isothermal and nonisothermal experiments [27,28]. In our case, a way to reconcile our results with the previous ones is to consider that, in the Pirnat et al. measurements, nucleation takes place only during induction time, then growth alone occurs afterwards ( $a=0, b=3, c=1 / 2$, then $n=1.5)$. Note also that the same little thermal effect on Avrami exponent has been observed by Pirnat et al., with $n$ varying from 1.4 to 1.7 with increasing temperature [18].

The other discrepancy with Pirnat et al. results concerns the reaction rate constant $k$, which they find almost independent upon temperature. The reason might also lie in the different thermal procedure, and the induction time they observe. The order of magnitude of $k$, around $2 \times 10^{-5} \mathrm{~s}^{-1}$ with our definition of $k$ (which corresponds to $k^{1 / n}$ in Pirnat et al.'s formula), is, however, comparable to ours at $810^{\circ} \mathrm{C}$.

Finally, note that both the $n$ Avrami exponent and activation energy we find for the pseudocubic-tetragonal phase transition of $\mathrm{Bi}_{3} \mathrm{NbO}_{7}$ are comparable to those measured in the devritrification transition of $\mathrm{PbGeO}_{3}$ $(n \sim 2.5$ and $E \sim 3.99 \mathrm{eV}[29])$.

\section{Electrical conductivity}

The complex impedance spectra measured at two different temperatures of 425 and $475^{\circ} \mathrm{C}$ for type-III $\mathrm{Bi}_{3} \mathrm{NbO}_{7}$ are presented in Fig. 11. They show the presence of two semicircles. The small arc at low frequency (see the enlarged inset) corresponds to a capacitance of the order of $10^{-7} \mathrm{~F}$ consistent with electrode polarization. We were not able to differentiate any grain boundary from bulk contribution within the large impedance arc at high frequency. The capacitance corresponding to this high frequency arc being around $10^{-11} \mathrm{~F}$, this contribution was interpreted as resulting from the bulk resistance of the sample. The resistance values were obtained by using a simple $\mathrm{R} / / \mathrm{CPE}$ model to fit the large arc of the impedance diagrams. The fitted phase angles are around $10^{\circ}$, indicating that semicircles are slightly depressed. The impedance spectra of the type-II phase are similar (not presented here).

In Fig. 12, the temperature dependence of total conductivity (ionic and electronic conductivity) for typeII and type-III $\mathrm{Bi}_{3} \mathrm{NbO}_{7}$ is given, in which a linear Arrhenius plot is observed for each phase. The slopes of the plots are determined as $1.06 \mathrm{eV}$ for the type-II phase and $0.80 \mathrm{eV}$ for type-III phase, respectively. In the whole temperature range, the tetragonal phase exhibits a higher electrical conductivity than that of the pseudocubic phase. This result is in good agreement with the conclusion speculated from RF dielectric measurements [17], where the tetragonal phase was found to exhibit a significantly

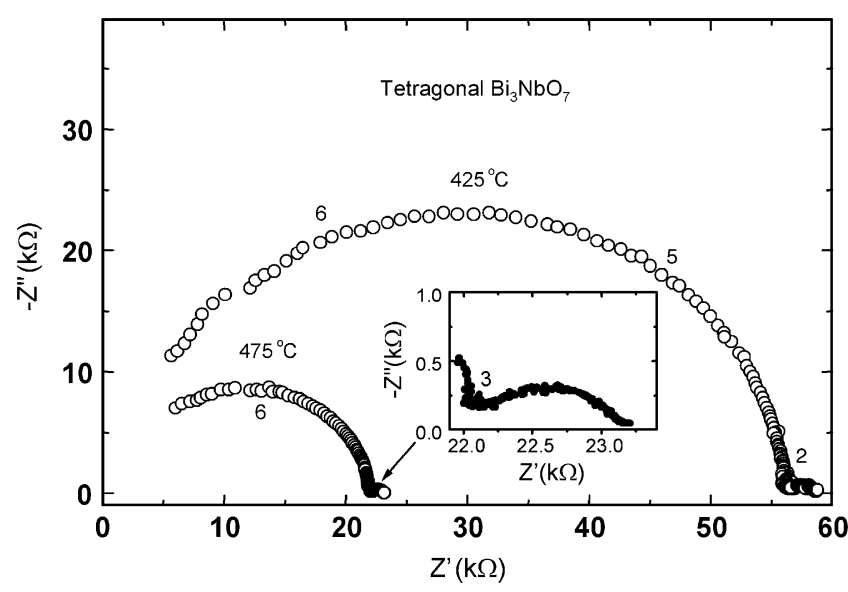

Fig. 11. Complex impedance spectra (Nyquist representation) of type-III $\mathrm{Bi}_{3} \mathrm{NbO}_{7}$ at two temperatures.

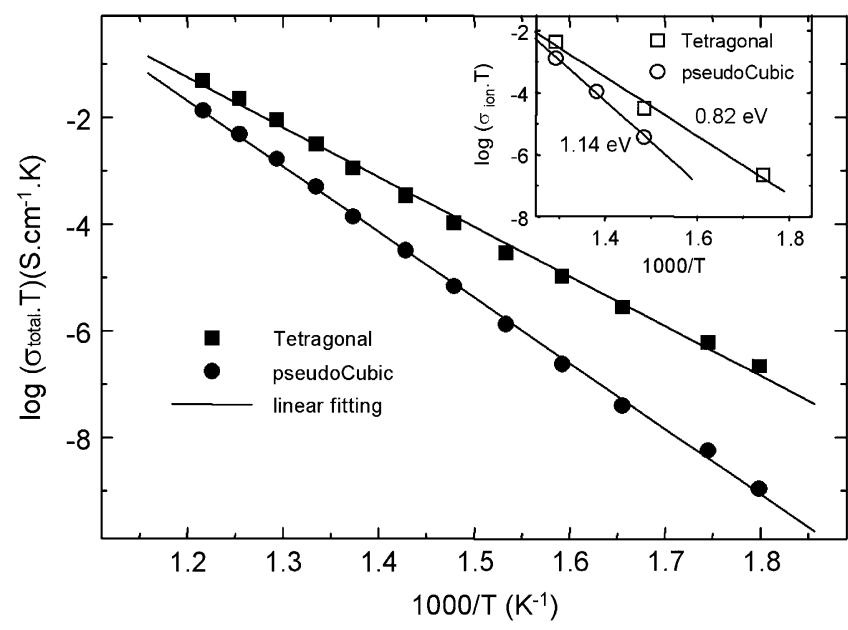

Fig. 12. Arrhenius plots of total electrical conductivity of tetragonal and pseudocubic $\mathrm{Bi}_{3} \mathrm{NbO}_{7}$. Inset: Arrhenius plots of ionic part of conductivity for both type-II and type-III phases. Note: Due to reactivity with $\mathrm{Pt}$ electrodes above $600{ }^{\circ} \mathrm{C}$, the thermal range is voluntarily limited. 
Table 2

Summary of electrical parameters of the pseudocubic and tetragonal $\mathrm{Bi}_{3} \mathrm{NbO}_{7}$ phases, showing ionic conductivity $\sigma_{\text {ion }}$, electronic conductivity $\sigma_{\mathrm{e}}$, and ionic transference number $t_{0}$

\begin{tabular}{|c|c|c|c|c|}
\hline \multirow[t]{2}{*}{ Temperature $\left({ }^{\circ} \mathrm{C}\right)$} & \multicolumn{2}{|l|}{ Pseudocubic (type-II) } & \multicolumn{2}{|l|}{ Tetragonal (type-III) } \\
\hline & $\sigma_{\text {ion }} / \sigma_{\mathrm{e}}\left(\times 10^{-5}\right)\left(\mathrm{S} \mathrm{cm}^{-1}\right)$ & $t_{0}$ & $\sigma_{\text {ion }} / \sigma_{\mathrm{e}}\left(\times 10^{-5}\right)\left(\mathrm{S} \mathrm{cm}^{-1}\right)$ & $t_{0}$ \\
\hline 500 & $7.20 / 0.81$ & 0.90 & $12.2 / 4.5$ & 0.73 \\
\hline 450 & $2.56 / 0.35$ & 0.88 & - & - \\
\hline 400 & $0.65 / 0.20$ & 0.76 & $1.65 / 1.1$ & 0.60 \\
\hline 300 & - & - & $0.21 / 0.13$ & 0.61 \\
\hline
\end{tabular}

higher high-temperature permittivity at $1 \mathrm{kHz}$ than that of the pseudocubic phase.

The Wagner polarization method has been used to evaluate the electronic part of conductivity in type-II and type-III $\mathrm{Bi}_{3} \mathrm{NbO}_{7}$ phases. The pure electronic and ionic conductivities at different temperatures for both phases are given in Table 2, together with the ionic transference numbers $t_{0}=1-\sigma_{\mathrm{e}} / \sigma_{\text {total }}$. It is found that both the ionic conductivity and electronic conductivity in the tetragonal phase are higher than those in the pseudocubic phase at a comparable temperature.

The plots of ionic conductivity versus reciprocal of temperature are adequately fitted by the Arrhenius law: $\sigma T=\sigma_{0} \exp \left(-E_{\mathrm{a}} / k T\right)$ (see the inset of Fig. 12), where $\sigma_{0}$ is the pre-exponential factor, $E_{\mathrm{a}}$ is the activation energy. From the slopes of the Arrhenius plots, the activation energies $\left(E_{\mathrm{a}}\right)$ of oxygen ion diffusion are determined as $1.14 \mathrm{eV}$ for the type-II phase and $0.82 \mathrm{eV}$ for the type-III phase. The lower diffusion activation energy of the tetragonal phase shows that the type-III $\mathrm{Bi}_{3} \mathrm{NbO}_{7}$ structure offers a lower energy barrier to oxide ion migration than the type-II structure. In comparison, the electronic activation energy is comparable in both phases, around $0.7 \mathrm{eV}$.

Although ionic conductivity is dominant in both the type-II and type-III phases, the relatively low ionic transference number demonstrates that the electronic conductivity, reported as p-type [8], is still substantial, especially at low temperature. Indeed, the value of ionic transference number is found to decrease with decreasing temperature in both phases, due to the smallest activation energy for electronic transport. The electronic conduction is suggested to originate from the partial oxidation of $\mathrm{Bi}^{3+}$ to $\mathrm{Bi}^{5+}$ [30].

From the thermal evolution of the unit cell volume of $\mathrm{Bi}_{3} \mathrm{NbO}_{7}$, a slight shrinkage of lattice induced by the pseudocubic-tetragonal transformation is observed (see Fig. 2). The higher conductivity observed in the phase with smaller unit cell volume implies that the difference in cell volume is not responsible for the difference of transport properties. Thus in a condition of same concentration of oxygen vacancies, the large difference of the conduction properties between type-II and type-III $\mathrm{Bi}_{3} \mathrm{NbO}_{7}$ should be attributed to the structural differences. Three main parameters can influence the anionic conduction in these phases: the cationic arrangement, the Bi lone electronic pairs and the location of oxygen vacancies. The $\mathrm{Bi}^{3+} / \mathrm{Nb}^{5+}$ distribution determines the interaction potential seen by oxide ions. Depending on its position and orientation, the bismuth lone pair can either block oxygen migration, or favor it through the polarization phenomenon. Finally, the oxygen vacancy distribution is determinant for migration path. All three parameters are different in the type-II and in the type-III phases, therefore implying a difference in the subtle resulting balance, which controls the carriers number and mobility as well as their migration activation energy.

\section{Conclusion}

The kinetics of the metastable pseudocubic to stable tetragonal phase transformation in $\mathrm{Bi}_{3} \mathrm{NbO}_{7}$ has been studied using isothermal in situ XRD. The obtained value of 2.5 for the Avrami exponent is consistent with a 3D diffusion-controlled transformation, with constant nucleation rate. While for the tetragonal-cubic transition at $940{ }^{\circ} \mathrm{C}$, the short reaction time of $20 \mathrm{~min}$ implies that the nucleation and growth of the transformation fulfills nearly instantaneously. Electrical measurements indicate that, although both the pseudocubic and tetragonal $\mathrm{Bi}_{3} \mathrm{NbO}_{7}$ exhibit a mixed ionic and electronic transport property, the tetragonal phase shows a higher electrical conductivity than the pseudocubic phase in the whole investigated temperature range. The reason is suggested to be associated with the redistribution of oxygen sublattice (or oxygen vacancies) induced by superstructural ordering in the tetragonal $\mathrm{Bi}_{3} \mathrm{NbO}_{7}$, which appears to increase the mobility of free charge carriers and therefore improves the electrical conductivity.

\section{Acknowledgments}

This work was supported by the CNRS-CAS convention (Project No. 14913). The authors acknowledge the Pays-dela-Loire Region for X.P. Wang's grant.

\section{References}

[1] T. Takahashi, H. Iwahara, Mater. Res. Bull. 13 (1978) 1447.

[2] N.M. Sammes, G.A. Tomopsett, H. Näfe, F. Aldinger, J. Eur. Ceram. Soc. 19 (1999) 1801. 
[3] H.A. Hrwig, A.G. Gerards, J. Solid State Chem. 26 (1978) 265.

[4] P. Shuk, H.-D. Wiemhöfer, U. Guth, W. Göpel, M. Greenblatt, Solid State Ionics 89 (1996) 179.

[5] A. Castro, E. Aguado, J.M. Rojo, P. Herrero, R. Enjalbert, J. Galy, Mater. Res. Bull. 33 (1998) 31.

[6] W. Zhou, J. Solid State Chem. 108 (1994) 381.

[7] C.D. Ling, J. Solid State Chem. 148 (1999) 380.

[8] A.A. Yaremchenko, V.V. Kharton, E.N. Naumovich, A.A. Vecher, J. Solid State Electrochem. 2 (1998) 146.

[9] C.S. Chen, B.A. Boukamp, H.J.M. Bouwmeester, G.Z. Cao, H. Kruidhof, A.J.A. Winnubst, A.J. Burgraaf, Solid State Ionics 76 (1995) 23.

[10] D. Hirabayashi, A. Hashimoto, T. Hibino, U. Harada, M. Sano, Electrochem. Solid State Lett. 7 (2004) A108.

[11] W. Zhou, D.A. Jefferson, J.M. Thomas, J. Solid State Chem. 70 (1987) 129.

[12] D. Tang, W. Zhou, J. Solid State Chem. 119 (1995) 311.

[13] C.D. Ling, R.L. Withers, S.S. Schmid, J.G. Thompson, J. Solid State Chem. 137 (1998) 42.

[14] R.L. Withers, C.D. Ling, S. Schmid, Z. Kristallogr. 214 (1999) 296.

[15] C.D. Ling, M. Johnson, J. Solid State Chem. 177 (2004) 1838.
[16] M. Valant, B. Jančar, U. Pirnat, D. Suvorov, J. Eur. Ceram. Soc. 25 (2005) 2829.

[17] M. Valant, D. Suvorov, J. Am. Ceram. Soc. 86 (2003) 939.

[18] U. Pirnat, M. Valant, B. Jancar, D. Suvorov, Chem. Mater. 17 (2005) 5155.

[19] J. Rodriguez-Carvajal, Physica B 192 (1993) 55.

[20] R. Miida, M. Tanaka, Jpn. J. Appl. Phys. 29 (1990) 1132.

[21] A.M. Fogg, S.J. Price, R.J. Francis, S. O'Brien, D. O'Hare, J. Mater. Chem. 10 (2000) 2355.

[22] M. Avrami, J. Chem. Phys. 7 (1939) 1103.

[23] M. Avrami, J. Chem. Phys. 8 (1940) 212.

[24] M. Avrami, J. Chem. Phys. 9 (1941) 177.

[25] S. Ranganathan, M. von Heimendahl, J. Mater. Sci. 16 (1981) 2401.

[26] G. Ruitenberg, A.K. Petford-Long, R.C. Doole, J. Appl. Phys. 92 (2002) 3116

[27] E.J. Mittemeijer, J. Mater. Sci. 27 (1992) 3977.

[28] J.R. Frade, V.V. Kharton, D. Marrero-López, P. Nuñez, J.C.C. Abrantes, Thermochim. Acta 435 (2005) 85.

[29] C. Tomasi, M. Scavini, A. Cavicchioli, A. Speghini, M. Bettinelli, Thermochim. Acta 432 (2005) 2.

[30] H. Mizoguchi, K. Ueda, H. Kawazoe, H. Hosono, T. Omata, S. Fujitsu, J. Mater. Chem. 7 (1997) 943. 